\title{
In utero delivery of miRNA induces epigenetic alterations and corrects pulmonary pathology in congenital diaphragmatic hernia
}

\author{
Sarah J Ullrich ${ }^{1}$, Nicholas K Yung ${ }^{1}$, Tory J Bauer-Pisani ${ }^{1}$, Nathan L Maassel ${ }^{1}$, Mary Elizabeth \\ Guerra $^{1}$, Mollie Freedman-Weiss ${ }^{1}$, Samantha L Ahle ${ }^{1}$, Adele S Ricciardi ${ }^{1,2}$, Maor Sauler ${ }^{3}$, W \\ Mark Saltzman $^{2,4,5,6}$, Alexandra S Piotrowski-Daspit ${ }^{2}$, David H. Stitelman ${ }^{1}$ \\ 1. Department of Surgery, Yale University, New Haven, CT, 06510, USA \\ 2. Department of Biomedical Engineering, Yale University, New Haven, CT, 06511, USA \\ 3. Department of Medicine, Yale University, New Haven, CT, 06510, USA \\ 4. Department of Chemical \& Environmental Engineering, Yale University, New Haven, \\ CT, 06511, USA \\ 5. Department of Cellular \& Molecular Physiology, Yale University, New Haven, CT, \\ 06511, USA \\ 6. Department of Dermatology, Yale University, New Haven, CT, 06510, USA
}

Correspondence should be addressed to S.J.U. (sarah.ullrich@yale.edu)

Sarah Ullrich

330 Cedar Street, FMB 107

New Haven, CT 06510

Telephone: +1 (631) 275-3851

Fax: +1 (203) 737-5209

Short Title: In utero delivery of miRNA 


\section{Abstract:}

Structural fetal diseases, such as congenital diaphragmatic hernia (CDH) can be diagnosed

34 prenatally. Neonates with $\mathrm{CDH}$ are healthy in utero as gas exchange is managed by the placenta,

35 but impaired lung function results in critical illness from the time a baby takes its first breath.

36 During fetal development, lungs are capable of remarkable growth and the fetus does not yet

37 require lung function for gas exchange. MicroRNA (miR) 200b and its downstream targets in the

38 TGF $\beta$ pathway are critically involved lung branching morphogenesis. Here we characterize the

39 expression of miR200b and the TGF $\beta$ pathway at different gestational times using a rat model of

$40 \mathrm{CDH}$. Fetal rats with $\mathrm{CDH}$ are deficient in miR200b at gestational day 18 . We demonstrate that

41 NPs loaded with miR200b given systemically to fetal rats result in changes in the TGF $\beta$ pathway;

42 these epigenetic changes improve lung size, lung morphology, and lung vascularization. This is

43 the first demonstration of in utero epigenetic therapy to improve lung growth and development in

44 a pre-clinical model. With refinement, this technique could be applied to fetal cases of CDH or 45 other forms of impaired lung development in a minimally invasive fashion. 


\section{Introduction}

Congenital Diaphragmatic Hernia $(\mathrm{CDH})$ results from incomplete fusion of the diaphragm

in utero causing the abdominal organs to herniate into the chest cavity. ${ }^{1,2}$ It is a relatively

49 common condition with an incidence of 1 in 2,000 to 3,000 live births. ${ }^{3,4}$ Even with modern neonatal critical care, the overall mortality is approximately $30 \%$, and approaches $80 \%$ for those

51 with severe disease. ${ }^{5,6}$ Infants with $\mathrm{CDH}$ have poorly developed lungs with two predominant

52 pathologic features: pulmonary hypoplasia $(\mathrm{PH})$ characterized by reduced distal airway

53 branching with fewer alveoli that are thick walled and persistent pulmonary hypertension (PPH)

54 due to hypermuscularized arterioles that are less vasoactive. ${ }^{7-10}$

There is a compelling rationale to develop fetal intervention for $\mathrm{CDH}$ given the potential to reverse pulmonary hypoplasia in utero, before the delivered fetus takes its first breath. Trials examining Fetoscopic Endoluminal Tracheal Occlusion (FETO), where a balloon is deployed in

58 the trachea under fetoscopic guidance and subsequently removed weeks later, have demonstrated

59 a survival benefit for those with severe disease. ${ }^{11,12}$ But FETO carries a substantial risk of

60 complications such as preterm premature rupture of the membranes and preterm delivery.

61 Among fetuses treated with FETO, those who had subsequent lung growth and survived after

62 balloon deployment and retrieval had higher miR200b expression in their tracheal fluid aspirates

63 than those who did not respond to tracheal occlusion. ${ }^{13}$ The microRNA 200 family inhibits

64 several genes in the TGF $\beta$ pathway, which has been previously demonstrated to modulate lung

65 branching during development, and in vitro studies suggest that treatment with miR200b can

66 increase branching morphogenesis. ${ }^{13-18}$ miRNAs are rapidly degraded by serum enzymes and intracellular RNases, making them

68 highly unstable in vivo. ${ }^{19}$ For miRNAs to be used therapeutically, it is essential for them to be 
69 delivered in a vehicle that confers high stability and avoids potential toxicities. ${ }^{20,21}$ A variety of

70 approaches have been explored to address these limitations, such as chemically modifying the

71 miRNA mimics to enhance stability or using viral vectors, lipid emulsions, liposomes, lipid

72 nanoparticles, or polymeric nanoparticles. ${ }^{19,22-29}$ But stabilized miRNA mimics have decreased

73 mRNA silencing ability, viral vectors have a poor safety profile, and cationic lipids are toxic to

74 cells and pro-inflammatory. ${ }^{19,30}$ Polymeric nanoparticles (NPs) can be used for the sustained

75 delivery of drugs, are non-toxic with a low side effect profile, and can be optimized to target

76 fetal lung. ${ }^{31-33}$ We have recently demonstrated that polymeric NPs can safely be used to deliver

77 editing reagents in the form of peptide nucleic acids (PNAs) and donor DNAs in utero to correct

78 a disease-causing mutation in the $\beta$-globin gene in a mouse model of human $\beta$-thalassemia. ${ }^{34}$

79 Optimization for delivery to fetal lung demonstrated that intravenous delivery led to improved

80 lung delivery over intraamniotic delivery, and that cationic poly(amine-co-ester) (PACE)

81 nanoparticles (NPs) were delivered most efficiently to fetal lung. ${ }^{33}$

82 Here, we sought to deliver miR200b in utero using PACE NPs to treat a rat model of CDH.

83 We found that in utero delivery of miR200b induces alterations in the TGF $\beta$ pathway, leads to

84 pulmonary vascular remodeling, and improves pulmonary hypoplasia. 


\section{Results}

miR200b expression and TGFBb signaling is altered during late gestation in the Nitrofen

Branching morphogenesis of fetal rat lung is completed during the canalicular stage of lung development (E18 through E20), whereas the terminal airspaces develop and divide during the

90 saccular stage (E21-P4). ${ }^{35}$ It has been previously demonstrated that miR200b regulates distal

91 airway branching, and that at E18 miR200b expression is reduced in nitrofen exposed lungs, and

92 at E21 miR200b expression is increased in nitrofen exposed lungs. ${ }^{17,18}$ To determine an ideal

93 therapeutic window for in utero miR delivery, we further elucidated the temporal expression of

94 miR200b in the wild-type (WT) and nitrofen-induced CDH lungs. The relative expression of

95 miR200b in WT and CDH lungs were quantified using RT-qPCR. In WT fetal rat lung, miR200b

96 levels are stable during the canalicular phase and undergo a 1.7-fold increase at the transition to

97 the saccular phase (Figure 1A). In CDH lungs, there is a $40 \%$ decrease in the relative expression

98 of miR200b at E18 during the early canalicular stage (Figure 1B). During the mid and late

99 canalicular phase, there is no difference in the relative expression of miR200b between WT and

100 CDH lungs. At E21, the relative expression of miR200b is 1.4-fold greater in CDH lungs

101 compared with WT lungs.

102 The TGF $\beta$ signaling pathway is a regulator of branching morphogenesis in pulmonary

103 development and is modulated by miR200b. ${ }^{36,37}$ The role of the TGF $\beta$ pathway in CDH is less

104 clearly defined. ${ }^{38}$ We found that in WT lungs, the expression of TGF $\beta$ and its downstream

105 targets increases as the lungs transition from the canalicular to the saccular phase (Figure 1C). In

$106 \mathrm{CDH}$ lungs, the expression of TGF $\beta$ and its downstream targets is increased during the

107 canalicular phase compared with WT lungs. In CDH lungs, the TGF $\beta$ pathway does not undergo 
108 the same increase in expression during late development, with the relative expression of most

109 downstream targets remaining stable between E19 and E21.

110 In utero delivery of miR200b induces epigenetic changes

111 Because we found that miR200b levels were reduced in CDH lungs at E18, we elected to target

112 this time point for treatment. Our previous data demonstrated that the majority of nucleic acid

113 release from PACE60 NPs occurs within the first 24 hours. ${ }^{39}$ To align delivery of miR200b with

114 the appropriate therapeutic window, we injected NPs at E17. To validate if in utero injection of

115 miRNA PACE60 NPs led to an increase in miR200b levels, WT fetuses were injected with

116 control or miR200b loaded NPs. Lungs were evaluated 4 hours after injection. Those injected

117 with miR200b had a 12 fold increase in miR200b levels (Figure 2A).

118 Previous in vitro studies demonstrated that treatment with a miR200b mimic abrogates nitrofen

119 induced upregulation of the TGF $\beta$ pathway. We treated nitrofen-exposed pups with control or

120 miR200b NPs at E17 and assessed the relative expression of the TGF $\beta$ pathway using RT-qPCR

121 at E19 and E21. We found that pups with CDH who were treated with miR200b PACE60 NPs

122 had a decrease in the relative expression of TGF $\beta 1$ and SMAD2 at E19 compared with pups

123 treated with control NPs (Figure 2B). At E21, pups treated with miR200b had a decrease in

124 TGF $\beta 1$ expression and SMAD3 expression compared to those treated with control NPs (Figure

125 2C). There were no instances where pups treated with control NPs had a change in gene

126 expression and miR200b PACE60 NPs did not.

\section{$127 \quad$ Lung morphology improves after in utero treatment with miR200b}

128 To assess if in utero treatment with miR200b lead to morphometric changes in fetal lung, lungs

129 of CDH pups were harvested at E21 after treatment with either control or miR200b PACE60 NPs

130 at E17. Treatment with miR200b improved pulmonary hypoplasia in pups with CDH (Figure 3 
131 A-C). The lung to body weight ratios of pups treated with miR200b NPs increased by $27 \%(2.2 \%$

132 vs $2.8 \%$ ), and approached the ratios of WT pups (3.4\%, Figure 3D). Pulmonary airspace

133 improved $140 \%$ in pups treated with miR200b, approaching that of WT pups, as measured by

134 mean linear intercept (WT $37 \mu \mathrm{M}$, control $11 \mu \mathrm{M}$, miR200b $26 \mu \mathrm{M}$, Figure 3E). Additionally, pups

135 treated with miR200b had a 63\% decrease in mean alveolar thickness, decreased beyond that of

136 wild type pups (WT $9 \mu \mathrm{M}$, control $16 \mu \mathrm{M}$, miR200b $6 \mu \mathrm{M}$, Figure $3 \mathrm{~F}$ ).

137 In utero treatment with miR200b also led to pulmonary vascular remodeling (Figure 4A-C).

138 The pulmonary arteries of pups injected with miR200b had a $23 \%$ decrease in percent medial

139 wall thicknesses compared with those treated with control NPs (37.3\% vs 48.5\%, Figure 4D).

140 The pulmonary arteries of pups treated with miR200b showed equivalent percent medial

141 thicknesses to WT pups (Figure 4D).

\section{Discussion}

In this study, we demonstrated that a single intravenous fetal dose of PACE60 NPs

144 loaded with miR200b improves lung development in a rat model of CDH. Specifically, delivery

145 of miR200b induced epigenetic changes in the TGF $\beta$ pathway during the canalicular and

146 saccular stages of pulmonary development. In utero treatment with miR200b lead to larger lungs

147 with more airspace and favorable pulmonary vascular remodeling at term in pups with CDH.

148 This targeted, minimally invasive in utero strategy has the potential to avoid substantial antenatal 149 morbidity and mortality in $\mathrm{CDH}$.

150 We examined the relative expression of miR200b during the canalicular and saccular stages

151 of rat lung development in both WT and CDH lungs and found that there is a marked deficiency

152 in miR200b at E18, followed by a rise in relative expression leading to overexpression at E21.

153 The relative expression of miR200b was equivalent at E19 and E20, suggesting the early 
154 canalicular phase is an optimal time for delivery of miR200b. $\mathrm{CDH}$ is a devastating lung disease

155 defined by impaired pulmonary vasculature and distal airway branching morphogenesis.

156 miR200b plays a key role in this process, promoting distal airway development by maintaining

157 an epithelial cell phenotype. ${ }^{17,18,36,40}$ The role of miR200b in the pathogenesis of CDH has been

158 less clearly delineated. Here, we defined a key role for miR200b in the pathogenesis of CDH.

159 Our findings are consistent with previous findings which examined miR200b expression in

160 nitrofen exposed lungs with varying degrees of hypoplasia. ${ }^{18}$

161 We demonstrated that in the nitrofen rat model, the TGF $\beta$ pathway is upregulated in the

162 lungs of pups with CDH relative to WT pups during the canalicular stage of lung development.

163 During the transition from the canalicular to saccular stages, the TGF $\beta$ pathway is upregulated as

164 branching morphogenesis slows down. However, in pups with $\mathrm{CDH}$, the expression of the TGF $\beta$

165 pathway does not change significantly, further indicating that the dynamics of the TGF $\beta$ pathway

166 is dysfunctional in the lungs of pups with CDH. The TGF $\beta$ pathway is one of many signal

167 transduction pathways regulating branching morphogenesis in the developing lung. TGF $\beta$

168 signaling is particularly important at the epithelial-mesenchymal interfaces during fetal

169 development and plays a key role in branching morphogenesis. ${ }^{16}$

170 In this study, we demonstrated that in utero treatment with miR200b can induce epigenetic

171 changes in the fetal lung. Though treatments designed to modify tumor epigenetics have long

172 been investigated for cancer, this has not been a focus of fetal interventions. ${ }^{41}$ Treatment

173 strategies that induce fetal epigenetic changes are particularly promising for CDH where - unlike

174 other congenital pulmonary diseases such as cystic fibrosis—a monogenic cause has not been

175 identified. ${ }^{42}$ This suggests that environmental, epigenetic factors play a substantial role in the

176 pathophysiology of $\mathrm{CDH}$. 
Fetal therapy for $\mathrm{CDH}$, before a baby takes a first breath, is the optimal time to improve lung

178 development as the lung is capable of structural change during fetal life and the gas exchange is

179 managed by the placenta. We envision that NP-based fetal epigenetic therapy could be delivered

180 through a small needle via ultrasound guided cannulation of the umbilical vessels, which has

181 been in clinical practice since the 1980 's and carries a low risk of fetal loss $(\sim 1 \%) .{ }^{43-45}$ Though

182 FETO has been demonstrated to have a survival benefit for those with severe disease, it is an

183 invasive procedure, and is associated with substantial risk of complications such as preterm

184 premature rupture of the membranes and preterm delivery. Another major limitation of FETO is

185 that while it induces lung growth, it does not improve pulmonary vascular remodeling and

186 pulmonary hypertension. ${ }^{46}$ In this study, we demonstrated that in utero treatment with miR $200 \mathrm{~b}$

187 PACE60 NPs leads to favorable pulmonary vascular remodeling, in addition to improving 188 pulmonary hypoplasia.

189 Though the PACE NPs used for this study have been optimized for delivery to fetal lung,

190 there is still the potential for off-target effects due to delivery to off target tissues. ${ }^{33}$ As this

191 therapy is translated to larger animal models, direct intra-tracheal administration could be

192 considered to minimize off-target delivery; intratracheal PACE vehicle delivery is well tolerated

193 in adult animals. ${ }^{47}$ We have demonstrated that miR200b delivery induces epigenetic changes in

194 the TGF $\beta$ pathway, but it also impacts other signal transduction pathways; further study is

195 warranted before translation to larger animal models. ${ }^{15}$ Success in such investigations could

196 provide a strong foundation for clinical translation of this novel therapeutic modality. 
Materials and Methods

\section{Polymer Synthesis and Nanoparticle Formulation}

PACE polymers were synthesized as described previously. ${ }^{39}$ Briefly, monomers (15pentadecanolide, $\mathrm{N}$-methyl diethanolamine, and diethyl sebacate) were dissolved in diphenyl ether with an enzyme lipase catalyst (Novozym 435). 60 mol\% PDL was used for the PACE polymer generated for this study. The synthesis reaction proceeded in two phases: an oligomerization phase for 18-20 hours under argon at 1 atm, and then a polymerization phase for 48-72 hours under vacuum. PACE polymers were characterized by NMR and GPC. PACE60 nanoparticles were formulated as described previously by double emulsion solvent evaporation using miR200b mimic (Invitrogen mirVana miRNA Mimic, hsa-miR-200b-3p, 5'UAAUACUGCCUGGUAAUGAUGAC -3', Cat\# 4464066, Ambion, Austin, TX) or a negative control miR (Invitrogen mirVana miRNA Mimic, Negative Control \#1, Cat\# 4464059, Ambion, Austin, TX). ${ }^{39,48}$ Characteristics of PACE60 NPs are displayed in Table 1.

\section{Rat Model and Injection}

All animal use was in accordance with the guidelines of the Animal Care and Use Committee of Yale University. CDH was modeled in rats as previously described. ${ }^{49}$ Time-dated pregnant Sprague-Dawley rats from Charles River Laboratories (Wilmington, MA) were gavage fed 100 mg of Nitrofen (Cat\# 33374, Sigma Aldrich, St. Louis, MO) dissolved in olive oil (Whole Foods, Austin, TX) on embryonic day E9. Time dated pregnant rats at 17 days post conception were anesthetized with inhaled isoflurane ( $2 \% \mathrm{vol} / \mathrm{vol}$ for induction and maintenance). Analgesia was provided with subcutaneous injection of $0.3 \mathrm{~mL}$ Buprenorphine $\mathrm{HCl}(0.015 \mathrm{mg} / \mathrm{mL})$ and $0.3 \mathrm{~mL}$ Meloxicam $(0.1 \mathrm{mg} / \mathrm{mL})$. The gravid uterus was exposed through a midline laparotomy incision. miR-loaded PACE60 NPs were resuspended by vortex and water 
220 bath sonication and delivered a dose of $30 \mathrm{mg} / \mathrm{kg}$ in $1 \times$ DPBS. NP suspension was drawn up

221 into a glass micropipette (tip diameter $\sim 60 \mu \mathrm{m}$ ) and injected intravascularly via vitelline vein of

222 each fetus using a pneumatic microinjector (Narishige; Japan). Rats were sacrificed at varying

223 time points post injection and fetuses were delivered by cesarean section.

\section{$224 R T-q P C R$}

225 Total miRNA from fetal rat lung was extracted using the mirVana miRNA Isolation Kit

226 (Cat\# AM1560, Ambion, Austin, TX, USA) according to the manufacturer's instructions. RT-

227 qPCR was performed for miRNA 200b and its mRNA downstream targets.

228 For the miRNA assay, 1-10 ng total RNA were reverse transcribed using the TaqMan

229 miRNA Assay for rno-miR200b-3p (rno481286_mir, Cat\# 4427975, Applied Biosystems, Foster

230 City, CA) TaqMan Advanced miRNA cDNA Synthesis Kit (Cat\# A28007, Applied Biosystems,

231 Foster City, CA) following the manufacturer's protocol as described. RT-qPCR was performed

232 using the TaqMan Fast Advanced Master Mix (Cat\# 4444556, Applied Biosystems, Foster City,

233 CA). U6 snRNA (Cat\# 4444556, Applied Biosystems, Foster City, CA) was used for

234 normalization.

235 RT-qPCR of mRNA was performed using the Applied Biosystems predesigned TaqMan

236 Gene Expression Assays (Cat\# 4331182, Applied Biosystems, Foster City, CA), and Superscript

237 IV VILO Master Mix (Cat\# 11766050, Invitrogen, Carlsbad, CA) per the manufacturer's

238 instructions for rat glyceraldehyde-3-phosphate dehydrogenase (Gapdh ; Rn01462662_g1),

239 transforming growth factor, beta 1 (Tgfb1; Rn00665219_g1), transforming growth factor, beta 2

240 (Tgfb1; Rn00579674_m1), zinc finger E-box binding homeobox 1 (Zeb1;Rn01538408_m1),

241 zinc finger E-box binding homeobox 2 (Zeb2 ; Rn01449758_m1), SMAD family member 2

242 (Smad2 ; Rn01527104_g1) and SMAD family member 3 (Smad3 ; Rn01422011_m1). 


\section{Lung Morphometry}

244 Fetal rats were delivered by cesarean section on E21 and lung: body weight ratio was

245 measured. For morphometric analysis, fetal lungs were fixed by intratracheal instillation of $4 \%$

246 paraformaldehyde under a constant pressure of $20 \mathrm{~cm} \mathrm{H}_{2} \mathrm{O}$. Visual confirmation of inflation was

247 obtained for all specimens analyzed. After ligation of the trachea, the lungs were immersed in

248 fixative and embedded in paraffin. Sections were stained with hematoxylin-eosin (H\&E) or

249 elastin van Gieson's (EvG) stain. The slides were imaged on a Zeiss Axio Scope light

250 microscope (Carl Zeiss Microscopy; Germany). Tissues were embedded in paraffin, and sliced

251 sections from medial, mid- and lateral lung zones were mounted onto slides for H\&E or EvG

252 staining. ${ }^{50}$

253 Airspace enlargement was measured by assessing mean linear intercept (Lm). Three random

254 fields were evaluated by microscopic projection and the alveolar size was estimated from the Lm

255 of the airspace as described previously. ${ }^{51-54}$ Alveolar thickness was measured as the mean septal

256 wall thickness of 10 terminal alveoli per field.

257 EvG stained sections were used for pulmonary arteriole (PA) remodeling assessment. The

258 PAs were distinguished from pulmonary veins based on their position and structure. In each

259 sample, only small PAs (external diameter: 20 to $100 \mu \mathrm{m}$ ) were used. External diameter (ED)

260 was measured across the shortest luminal profile between the external elastic laminae. Diameter

261 across the longest luminal profile also was measured, and only arteries in which the longest

262 diameter did not exceed ED by more than 50\% were used. Medial thickness was measured on the

263 same profile as ED. To assess PA remodeling, the percentage of the medial wall thickness was

264 calculated according to the following formula: $(2 \times$ medial wall thickness/external diameter $) \times$

$265100 \%$ as previously described. ${ }^{55-57}$ 


\section{Statistical Analysis}

267 Data are presented as means \pm S.D. unless otherwise noted and compared using student's t-

268 test, or one-way or two-way ANOVA followed by Tukey’s post-hoc test for multiple

269 comparisons when appropriate. Bonferroni correction was used to correct for multiple

270 comparisons. Statistical analyses were carried out using GraphPad Prism v9. A $P$ value of less

271 than 0.05 was considered statistically significant. 


\section{Acknowledgements}

This work was supported by the National Institutes of Health (UG3- HL147352 to W.M.S., K08HL135402 to M.S.). A.S.P. was supported by two NIH National Research Service Awards (NRSAs) (a T32 GM86287 training grant and a F32 HL142144 individual postdoctoral fellowship), as well as a postdoctoral research fellowship award (PIOTRO20F0) from the Cystic Fibrosis Foundation (CFF). S.J.U. was supported by a Cystic Fibrosis Foundation award (STITEL17G0). S.L.A was supported by a Yale Department of Surgery Ohse Award.

Authors S.J.U., W.M.S., A.S.R., D.H.S., and A.S.P. have filed an invention disclosure for the technology used in this manuscript with the Yale Office of Cooperative Research.

Authors W.M.S., A.S.R., D.H.S., A.S.P. are listed as inventors on the following patent: https://patents.justia.com/patent/20200113821

The graphical abstract for this manuscript was created using Mind The Graph:

https://mindthegraph.com/ 


\section{Author Contributions}

290 S.J.U. - conceptualization, data curation, formal analysis, investigation, methodology, project

291 administration, visualization, writing - original draft, writing - review \& editing

292 N.K.Y. - investigation, project administration, visualization

293 T.J.P. - data curation, investigation

294 N.L.M. - investigation

295 M.E.G. - formal analysis

296 M.F.W. - investigation

297 S.L.A - investigation, funding acquisition

298 A.S.R. - conceptualization, methodology, supervision

299 M.S. - formal analysis, funding acquisition, methodology, supervision, writing - review \&

300 editing

301 W.M.S. - conceptualization, funding acquisition, methodology, resources, supervision, writing -

302 review \& editing

303 A.S.P. - conceptualization, funding acquisition, investigation, methodology, supervision, writing

$304-$ review \& editing

305 D.H.S. - conceptualization, funding acquisition, methodology, project administration, resources,

306 supervision, writing - review \& editing 


\section{References}

3091 Longoni, M., High, F. A., Russell, M. K., Kashani, A., Tracy, A. A., Coletti, C. M., Hila, 310 R., Shamia, A., Wells, J., Ackerman, K. G. et al. Molecular pathogenesis of congenital diaphragmatic hernia revealed by exome sequencing, developmental data, and bioinformatics. Proc Natl Acad Sci U S A 111, 12450-12455, doi:10.1073/pnas.1412509111 (2014).

2 Veenma, D. C., de Klein, A. \& Tibboel, D. Developmental and genetic aspects of congenital diaphragmatic hernia. Pediatr Pulmonol 47, 534-545, doi:10.1002/ppul.22553 (2012).

3 Langham, M. R., Kays, D. W., Ledbetter, D. J., Frentzen, B., Sanford, L. L. \& Richards, D. S. Congenital Diaphragmatic Hernia: Epidemiology and Outcome. Clinics in Perinatology 23, 671-688, doi:https://doi.org/10.1016/S0095-5108(18)30201-X (1996).

4 Wynn, J., Krishnan, U., Aspelund, G., Zhang, Y., Duong, J., Stolar, C. J., Hahn, E., Pietsch, J., Chung, D., Moore, D. et al. Outcomes of congenital diaphragmatic hernia in the modern era of management. J Pediatr 163, 114-119 e111, doi:10.1016/j.jpeds.2012.12.036 (2013).

5 Coughlin, M. A., Werner, N. L., Gajarski, R., Gadepalli, S., Hirschl, R., Barks, J., Treadwell, M. C., Ladino-Torres, M., Kreutzman, J. \& Mychaliska, G. B. Prenatally diagnosed severe CDH: mortality and morbidity remain high. J Pediatr Surg 51, 10911095, doi:10.1016/j.jpedsurg.2015.10.082 (2016).

6 McGivern, M. R., Best, K. E., Rankin, J., Wellesley, D., Greenlees, R., Addor, M. C., Arriola, L., de Walle, H., Barisic, I., Beres, J. et al. Epidemiology of congenital diaphragmatic hernia in Europe: a register-based study. Arch Dis Child Fetal Neonatal Ed 100, F137-144, doi:10.1136/archdischild-2014-306174 (2015).

7 Ameis, D., Khoshgoo, N. \& Keijzer, R. Abnormal lung development in congenital diaphragmatic hernia. Seminars in Pediatric Surgery 26, 123-128, doi:https://doi.org/10.1053/j.sempedsurg.2017.04.011 (2017).

8 Donahoe, P. K., Longoni, M. \& High, F. A. Polygenic Causes of Congenital Diaphragmatic Hernia Produce Common Lung Pathologies. The American Journal of Pathology 186, 2532-2543, doi:https://doi.org/10.1016/j.ajpath.2016.07.006 (2016).

9 George, D. K., Cooney, T. P., Chiu, B. K. \& Thurlbeck, W. M. Hypoplasia and Immaturity of the Terminal Lung Unit (Acinus) in Congenital Diaphragmatic Hernia. American Review of Respiratory Disease 136, 947-950, doi:10.1164/ajrccm/136.4.947 (1987).

10 Kool, H., Mous, D., Tibboel, D., de Klein, A. \& Rottier, R. J. Pulmonary vascular development goes awry in congenital lung abnormalities. Birth Defects Research Part C: Embryo Today: Reviews 102, 343-358, doi:10.1002/bdrc.21085 (2014).

11 Deprest, J. A., Nicolaides, K. H., Benachi, A., Gratacos, E., Ryan, G., Persico, N., Sago, H., Johnson, A., Wielgoś, M., Berg, C. et al. Randomized Trial of Fetal Surgery for Severe Left Diaphragmatic Hernia. The New England journal of medicine, doi:10.1056/NEJMoa2027030 (2021). 
13 Pereira-Terra, P., Deprest, J. A., Kholdebarin, R., Khoshgoo, N., DeKoninck, P., Munck, A. A., Wang, J., Zhu, F., Rottier, R. J., Iwasiow, B. M. et al. Unique Tracheal Fluid

14 Burk, U., Schubert, J., Wellner, U., Schmalhofer, O., Vincan, E., Spaderna, S. \&

363

19 Rupaimoole, R. \& Slack, F. J. MicroRNA therapeutics: towards a new era for the doi:10.1038/nrd.2016.246 (2017). principles, expectations, and challenges. Chin J Cancer 30, 368-370, doi:10.5732/cjc.011.10186 (2011).

21 Li, Z. \& Rana, T. M. Therapeutic targeting of microRNAs: current status and future challenges. Nat Rev Drug Discov 13, 622-638, doi:10.1038/nrd4359 (2014).

22 van Rooij, E. \& Kauppinen, S. Development of microRNA therapeutics is coming of age. EMBO Mol Med 6, 851-864, doi:10.15252/emmm.201100899 (2014).

23 Blum, J. S. \& Saltzman, W. M. High loading efficiency and tunable release of plasmid DNA encapsulated in submicron particles fabricated from PLGA conjugated with poly-Llysine. J Control Release 129, 66-72, doi:10.1016/j.jconrel.2008.04.002 (2008).

24 Trang, P., Wiggins, J. F., Daige, C. L., Cho, C., Omotola, M., Brown, D., Weidhaas, J. B., Bader, A. G. \& Slack, F. J. Systemic delivery of tumor suppressor microRNA mimics using a neutral lipid emulsion inhibits lung tumors in mice. Mol Ther 19, 1116-1122, doi:10.1038/mt.2011.48 (2011). delivery of siRNA in cancer. J Intern Med 267, 44-53, doi:10.1111/j.13652796.2009.02191.x (2010). 
26 Joshi, H. P., Subramanian, I. V., Schnettler, E. K., Ghosh, G., Rupaimoole, R., Evans, C., Saluja, M., Jing, Y., Cristina, I., Roy, S. et al. Dynamin 2 along with microRNA-199a Acad Sci U S A 111, 5331-5336, doi:10.1073/pnas.1317242111 (2014).

27 Dahlman, J. E., Barnes, C., Khan, O. F., Thiriot, A., Jhunjunwala, S., Shaw, T. E., Xing, Y., Sager, H. B., Sahay, G., Speciner, L. et al. In vivo endothelial siRNA delivery using polymeric nanoparticles with low molecular weight. Nature Nanotechnology 9, 648-655, doi:10.1038/nnano.2014.84 (2014).

28 Woodrow, K. A., Cu, Y., Booth, C. J., Saucier-Sawyer, J. K., Wood, M. J. \& Mark Saltzman, W. Intravaginal gene silencing using biodegradable polymer nanoparticles densely loaded with small-interfering RNA. Nature Materials 8, 526-533, doi:10.1038/nmat2444 (2009).

29 Piotrowski-Daspit, A. S., Kauffman, A. C., Bracaglia, L. G. \& Saltzman, W. M. Polymeric vehicles for nucleic acid delivery. Advanced drug delivery reviews 156, 119 132, doi:10.1016/j.addr.2020.06.014 (2020).

30 Lv, H., Zhang, S., Wang, B., Cui, S. \& Yan, J. Toxicity of cationic lipids and cationic polymers in gene delivery. Journal of Controlled Release 114, 100-109, doi:https://doi.org/10.1016/j.jconrel.2006.04.014 (2006).

31 Makadia, H. K. \& Siegel, S. J. Poly Lactic-co-Glycolic Acid (PLGA) as Biodegradable Controlled Drug Delivery Carrier. Polymers (Basel) 3, 1377-1397, doi:10.3390/polym3031377 (2011).

32 Zhou, J., Liu, J., Cheng, C. J., Patel, T. R., Weller, C. E., Piepmeier, J. M., Jiang, Z. \& Saltzman, W. M. Biodegradable poly(amine-co-ester) terpolymers for targeted gene delivery. Nature Materials 11, 82-90, doi:10.1038/nmat3187 (2012).

33 Ullrich, S. J., Freedman-Weiss, M., Ahle, S., Mandl, H. K., Piotrowski-Daspit, A. S., Roberts, K., Yung, N., Maassel, N., Bauer-Pisani, T., Ricciardi, A. S. et al. Nanoparticles for delivery of agents to fetal lungs. Acta biomaterialia 123, 346-353, doi:10.1016/j.actbio.2021.01.024 (2021).

Ricciardi, A. S., Bahal, R., Farrelly, J. S., Quijano, E., Bianchi, A. H., Luks, V. L., Putman, R., Lopez-Giraldez, F., Coskun, S., Song, E. et al. In utero nanoparticle delivery for site-specific genome editing. Nat Commun 9, 2481, doi:10.1038/s41467-018-04894-2 (2018).

35 Schittny, J. C. Development of the lung. Cell Tissue Res 367, 427-444, doi:10.1007/s00441-016-2545-0 (2017).

36 Alejandre-Alcázar, M. A., Michiels-Corsten, M., Vicencio, A. G., Reiss, I., Ryu, J., de Krijger, R. R., Haddad, G. G., Tibboel, D., Seeger, W., Eickelberg, O. et al. TGF-beta signaling is dynamically regulated during the alveolarization of rodent and human lungs. Developmental dynamics : an official publication of the American Association of Anatomists 237, 259-269, doi:10.1002/dvdy.21403 (2008).

37 Chen, H., Sun, J., Buckley, S., Chen, C., Warburton, D., Wang, X. F. \& Shi, W. Abnormal mouse lung alveolarization caused by Smad3 deficiency is a developmental antecedent of centrilobular emphysema. American journal of physiology. Lung cellular and molecular physiology 288, L683-691, doi:10.1152/ajplung.00298.2004 (2005).

38 Mous, D. S., Buscop-van Kempen, M. J., Wijnen, R. M. H., Tibboel, D., Morty, R. E. \& Rottier, R. J. Opposing Effects of TGF $\beta$ and BMP in the Pulmonary Vasculature in 
Congenital Diaphragmatic Hernia. Frontiers in medicine 8, 642577, doi:10.3389/fmed.2021.642577 (2021).

39 Kauffman, A. C., Piotrowski-Daspit, A. S., Nakazawa, K. H., Jiang, Y., Datye, A. \& Saltzman, W. M. Tunability of Biodegradable Poly(amine- co-ester) Polymers for Customized Nucleic Acid Delivery and Other Biomedical Applications. Biomacromolecules 19, 3861-3873, doi:10.1021/acs.biomac.8b00997 (2018).

40 Chinoy, M. R. Lung growth and development. Frontiers in bioscience : a journal and virtual library 8, d392-415, doi:10.2741/974 (2003).

41 Bates, S. E. Epigenetic Therapies for Cancer. The New England journal of medicine 383, 650-663, doi:10.1056/NEJMra1805035 (2020).

42 Wagner, R., Montalva, L., Zani, A. \& Keijzer, R. Basic and translational science advances in congenital diaphragmatic hernia. Seminars in perinatology 44, 151170, doi:10.1053/j.semperi.2019.07.009 (2020).

43 Van Kamp, I. L., Klumper, F. J., Oepkes, D., Meerman, R. H., Scherjon, S. A., Vandenbussche, F. P. \& Kanhai, H. H. Complications of intrauterine intravascular transfusion for fetal anemia due to maternal red-cell alloimmunization. American journal of obstetrics and gynecology 192, 171-177, doi:10.1016/j.ajog.2004.06.063 (2005).

44 Bang, J., Bock, J. E. \& Trolle, D. Ultrasound-guided fetal intravenous transfusion for severe rhesus haemolytic disease. Br Med J (Clin Res Ed) 284, 373-374 (1982).

45 Pasman, S. A., Claes, L., Lewi, L., Van Schoubroeck, D., Debeer, A., Emonds, M., Geuten, E., De Catte, L. \& Devlieger, R. Intrauterine transfusion for fetal anemia due to red blood cell alloimmunization: 14 years experience in Leuven. Facts, views \& vision in ObGyn 7, 129-136 (2015).

46 Perrone, E. E. \& Deprest, J. A. Fetal endoscopic tracheal occlusion for congenital diaphragmatic hernia: a narrative review of the history, current practice, and future directions. Translational pediatrics 10, 1448-1460, doi:10.21037/tp-20-130 (2021).

47 Grun, M. K., Suberi, A., Shin, K., Lee, T., Gomerdinger, V., Moscato, Z. M., PiotrowskiDaspit, A. S. \& Saltzman, W. M. PEGylation of poly(amine-co-ester) polyplexes for tunable gene delivery. Biomaterials 272, 120780, doi:10.1016/j.biomaterials.2021.120780 (2021).

48 Cui, J., Piotrowski-Daspit, A. S., Zhang, J., Shao, M., Bracaglia, L. G., Utsumi, T., Seo, Y. E., DiRito, J., Song, E., Wu, C. et al. Poly(amine-co-ester) nanoparticles for effective Nogo-B knockdown in the liver. J Control Release 304, 259-267, doi:10.1016/j.jconrel.2019.04.044 (2019).

49 Kluth, D., Kangah, R., Reich, P., Tenbrinck, R., Tibboel, D. \& Lambrecht, W. Nitrofeninduced diaphragmatic hernias in rats: an animal model. J Pediatr Surg 25, 850-854, doi:10.1016/0022-3468(90)90190-k (1990).

50 Burgos, C. M., Pearson, E. G., Davey, M., Riley, J., Jia, H., Laje, P., Flake, A. W. \& Peranteau, W. H. Improved pulmonary function in the nitrofen model of congenital diaphragmatic hernia following prenatal maternal dexamethasone and/or sildenafil. Pediatric research 80, 577-585, doi:10.1038/pr.2016.127 (2016).

51 Knudsen, L., Weibel, E. R., Gundersen, H. J., Weinstein, F. V. \& Ochs, M. Assessment of air space size characteristics by intercept (chord) measurement: an accurate and efficient stereological approach. J Appl Physiol (1985) 108, 412-421, doi:10.1152/japplphysiol.01100.2009 (2010). 
52 Thurlbeck, W. M. Internal surface area and other measurements in emphysema. Thorax 22, 483-496, doi:10.1136/thx.22.6.483 (1967).

53 Kim, S. J., Shan, P., Hwangbo, C., Zhang, Y., Min, J. N., Zhang, X., Ardito, T., Li, A., epigenetic suppression of p16(INK4a). Aging Cell 18, e12914, doi:10.1111/acel.12914 (2019).

54 Hsia, C. C., Hyde, D. M., Ochs, M. \& Weibel, E. R. An official research policy statement of the American Thoracic Society/European Respiratory Society: standards for

56 Umeda, S., Miyagawa, S., Fukushima, S., Oda, N., Saito, A., Sakai, Y., Sawa, Y. \& quantitative assessment of lung structure. American journal of respiratory and critical care medicine 181, 394-418, doi:10.1164/rccm.200809-1522ST (2010).

55 Kanai, M., Kitano, Y., von Allmen, D., Davies, P., Adzick, N. S. \& Flake, A. W. Fetal tracheal occlusion in the rat model of nitrofen-induced congenital diaphragmatic hernia: tracheal occlusion reverses the arterial structural abnormality. J Pediatr Surg 36, 839Okuyama, H. Enhanced Pulmonary Vascular and Alveolar Development via Prenatal Administration of a Slow-Release Synthetic Prostacyclin Agonist in Rat Fetal Lung Hypoplasia. PLoS One 11, e0161334-e0161334, doi:10.1371/journal.pone.0161334 (2016). remodeling in a rat model induced by hypoxia and indomethacin. J Matern Fetal Neonatal Med 24, 172-182, doi:10.3109/14767058.2010.482608 (2011). 
bioRxiv preprint doi: https://doi.org/10.1101/2022.02.27.482144; this version posted March 1, 2022. The copyright holder for this preprint (which was not certified by peer review) is the author/funder. All rights reserved. No reuse allowed without permission.

511 Table 1. Characterization Data for PACE60 NP formulations.

512

\begin{tabular}{|l|c|c|c|}
\hline \multicolumn{1}{|c|}{ Formulation } & Diameter (nm) & $\begin{array}{c}\text { Polydispersity Index } \\
\text { (PDI) }\end{array}$ & Zeta Potential (mV) \\
\hline $\begin{array}{l}\text { PACE60 control } \\
\text { miRNA }\end{array}$ & $301 \pm 3$ & 0.170 & $30.2 \pm 0.1$ \\
\hline $\begin{array}{l}\text { PACE60 } \\
\text { miR200b }\end{array}$ & $323 \pm 2$ & 0.190 & $31.2 \pm 0.2$ \\
\hline
\end{tabular}

513 
Figure 1: miR200b and the TGF $\beta$ pathway expression during the canalicular and saccular phases of lung development in the Nitrofen rat model of $\mathrm{CDH}$. A) Bar graph showing the relative abundance of miR200b in wild type and $\mathrm{CDH}$ lungs during late gestation. All values normalized to wild-type at each day. Students t-test, $* * * * \mathrm{p}<0.0001$, Data are shown as mean \pm S.D. of $\mathrm{n}=$ 10-12. B) Relative expression of miR200b during late gestation in wild type and Nitrofen induced $\mathrm{CDH}$ fetal rat lungs during the canalicular and saccular phases of pulmonary development normalized to wild-type E19 lungs. Data shown as mean \pm S.D. of $n=10-12$ C) Heatmap of the relative expression of the TGF $\beta$ pathway during the canalicular (E19) and saccular (E21) phases of pulmonary development in WT and CDH lungs. Red = downregulated, green $=$ upregulated. $\mathrm{n}=4$.

Figure 2: In utero delivery of miR200b by PACE60 NPs alters miR200b expression levels and induces epigenetic changes in the TGF $\beta$ pathway. A) Bar graph showing the relative abundance of miR200b in WT fetal lungs at E17 4 hours post injection with control and miR200b PACE60 NPs. Values normalized to lungs injected with control miRNA PACE NPs. Students t-test,**** $\mathrm{p}<0.0001$, Data are shown as mean \pm S.D. of $n=3$. B) Bar graph showing the relative abundance of the downstream targets of the TGF $\beta$ pathway in WT fetal lungs and those injected with control miRNA and miR200b PACE60 NPs at E19. Values normalized to WT lungs. 2-Way ANOVA, $* \mathrm{p}<0.05, * * \mathrm{p}<0.01, * * * \mathrm{p}<0.001, * * * * \mathrm{p}<0.0001$, Data are shown as mean \pm S.D. of $n=4-6$. C) Bar graph showing the relative abundance of the downstream targets of the TGF $\beta$ pathway in WT fetal lungs and those injected with control miRNA and miR200b PACE60 NPs at E21. Values normalized to WT lungs. 2-Way ANOVA, **p<0.01, **** $\mathrm{p}<0.0001$, Data are shown as mean \pm S.D. of $n=4-6$.

Figure 3: In utero delivery of miR200b PACE60 NPs improves pulmonary hypoplasia in the Nitrofen Rat model of CDH Representative hematoxylin and eosin images of A) Term WT lungs. B) Term CDH lungs treated with Control miRNA PACE60 NPs and C) Term CDH lungs treated with miR200b PACE60 NPs. Scale bars $=100 \mu \mathrm{m}$. D) Lung to body weight ratios of WT lungs, CDH lungs treated with control miRNA PACE60 NPs and CDH lungs treated with miR200b PACE60 NPs. Each data point represents a single pup. 1-Way ANOVA, **p<0.01, *** $\mathrm{p}<0.001, * * * * \mathrm{p}<0.0001$. E) Mean linear intercept of WT lungs, CDH lungs treated with control miRNA PACE60 NPs and CDH lungs treated with miR200b PACE60 NPs. Each data point represents the lung of a single pup. 1-Way ANOVA, **** $\mathrm{p}<0.0001$. F) Mean alveolar thickness of WT lungs, CDH lungs treated with control miRNA PACE60 NPs and CDH lungs treated with miR200b PACE60 NPs. 1-Way ANOVA, **p<0.01, *** $\mathrm{p}<0.001, * * * * \mathrm{p}<0.0001$.

Figure 4: In utero delivery of miR200b PACE60 NPs results in pulmonary vascular remodeling in the Nitrofen Rat model of CDH. Representative images of Elastin van Gieson stained pulmonary arteries from A) Term WT lungs B) Term CDH lungs treated with control miRNA PACE60 NPs and C) Term CDH lungs treated with miR200b PACE60 NPs. Scale bars $=20 \mu \mathrm{m}$. D) Percent medial wall thickness of WT lungs, CDH lungs treated with control miRNA PACE60 NPs and CDH lungs treated with miR200b PACE60 NPs. Each data point represents an individual arteriole. 1-Way ANOVA, $* * \mathrm{p}<0.01, * * * * \mathrm{p}<0.0001$. 
A.

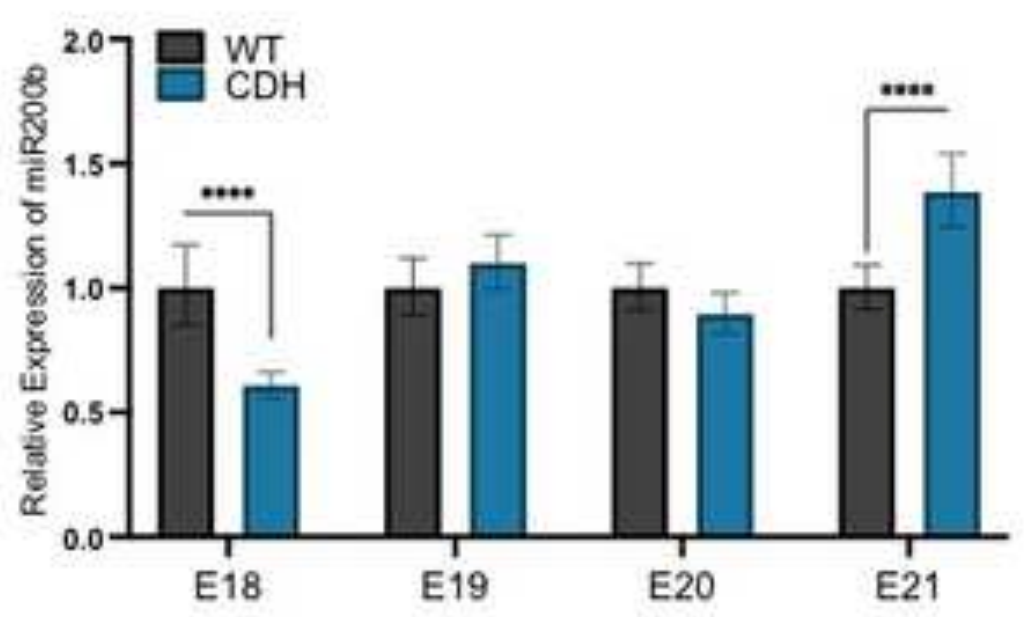

C.

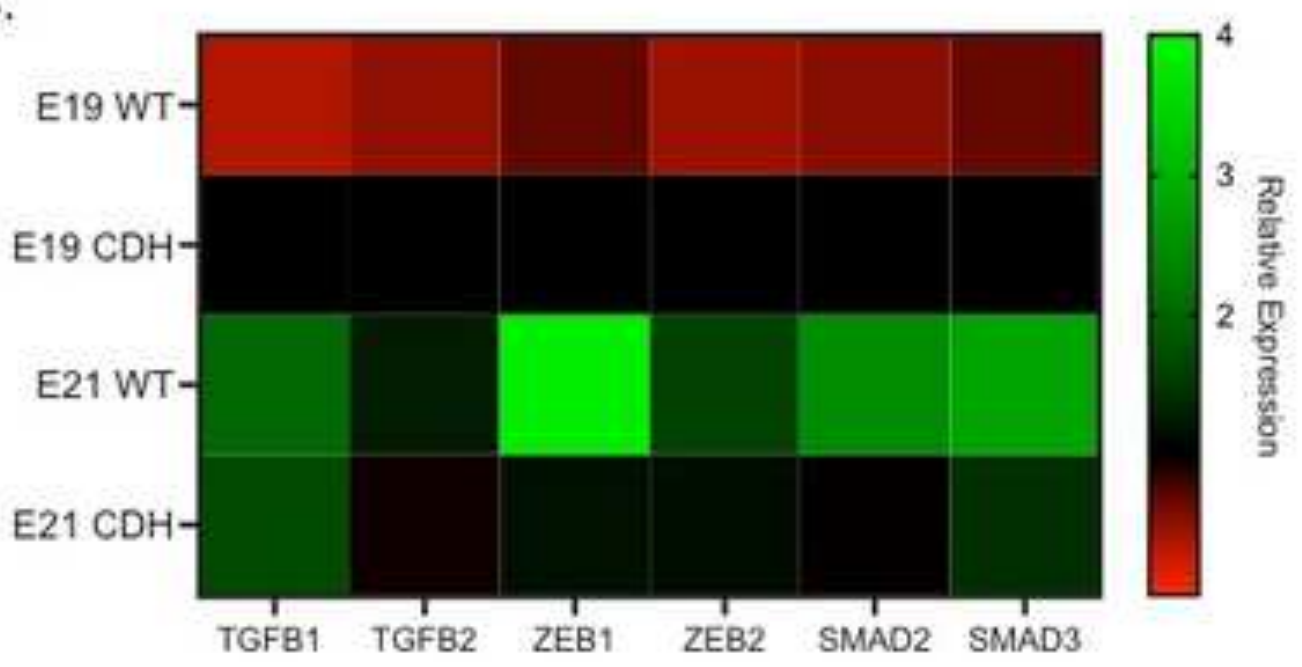

B.

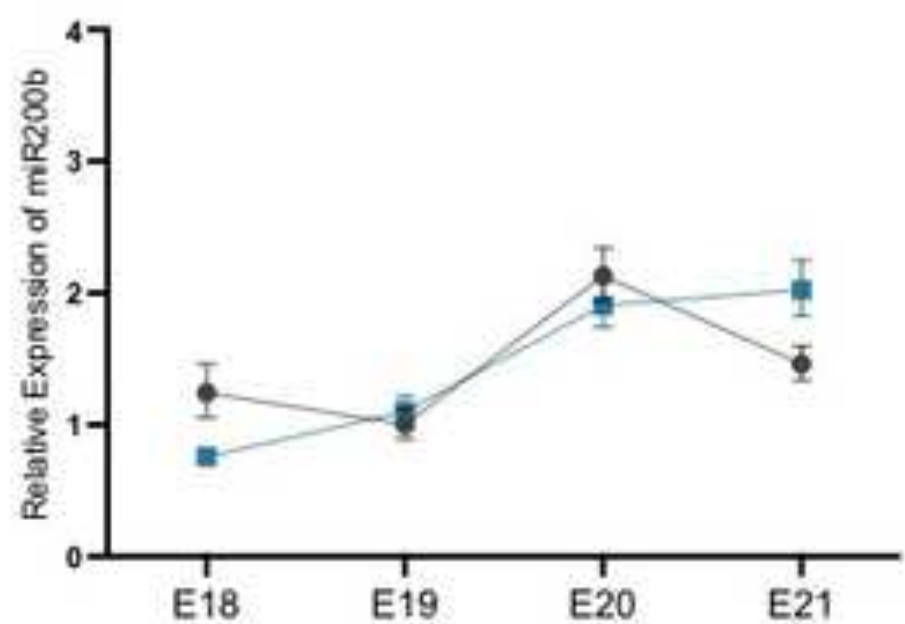

- WT

- $\mathrm{CDH}$

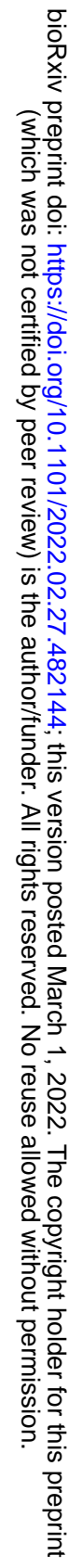



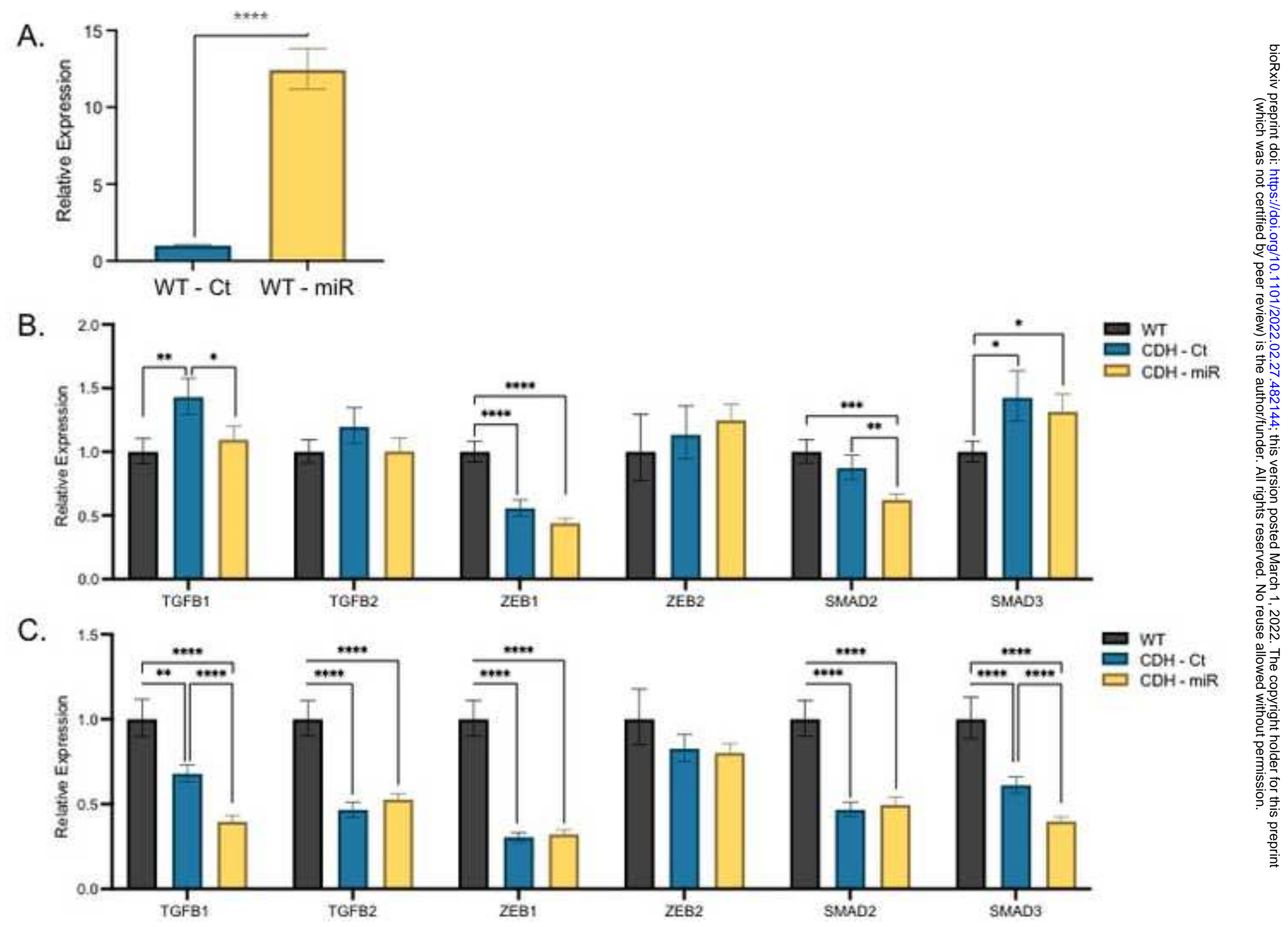


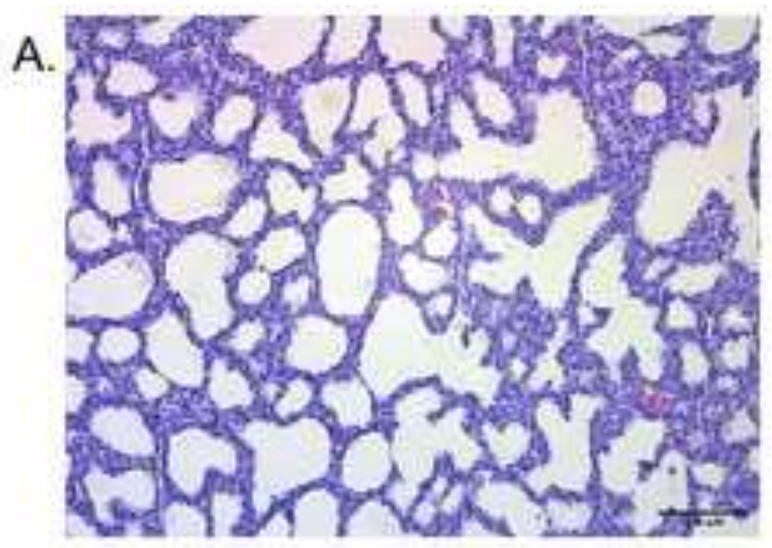

B.

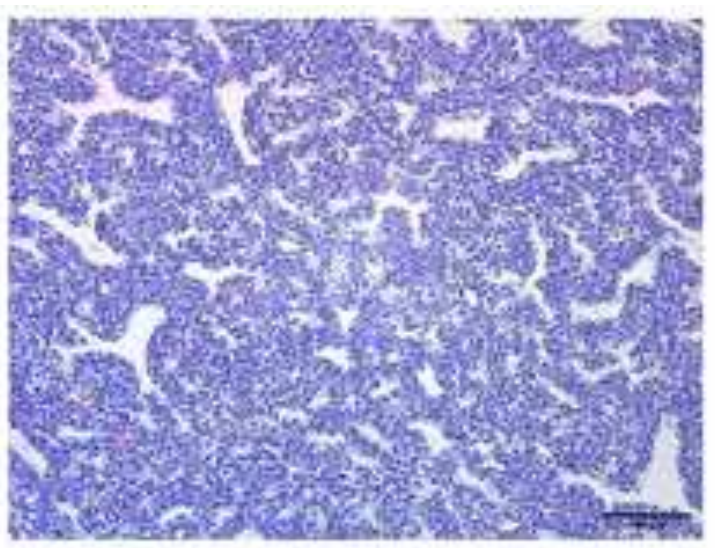

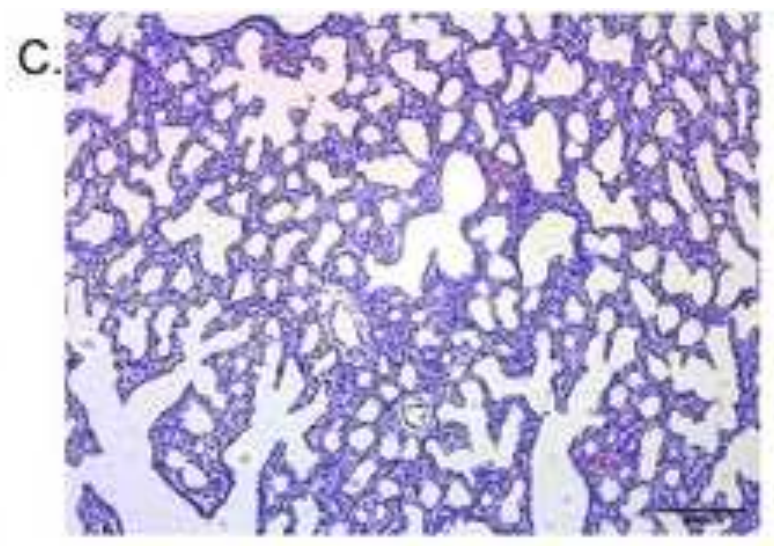

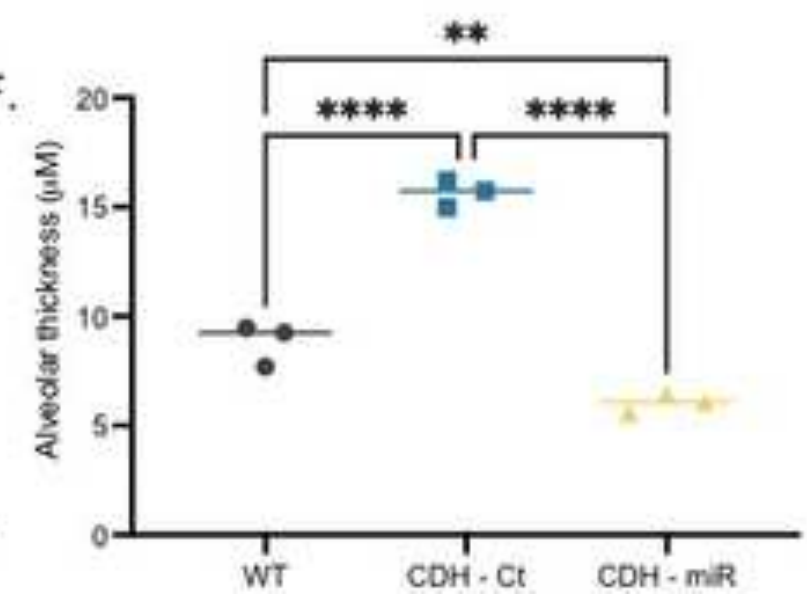

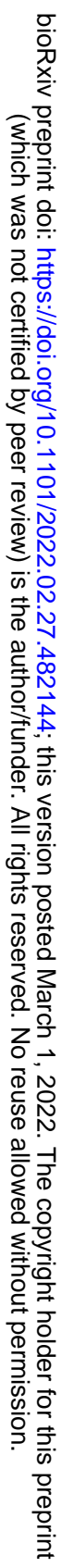

D.

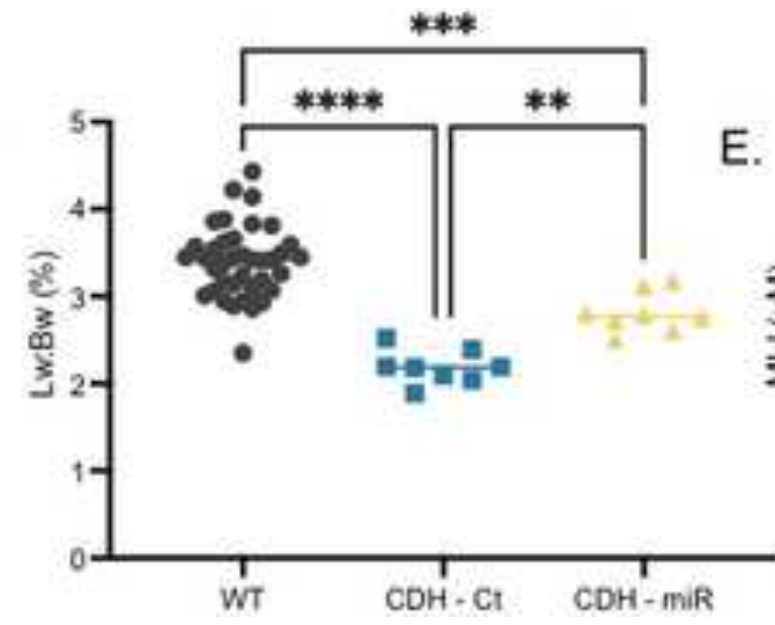

*1013.

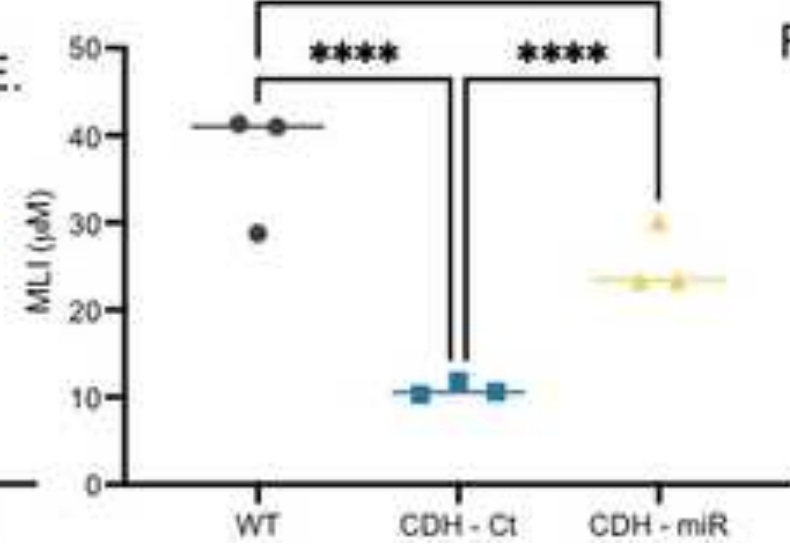




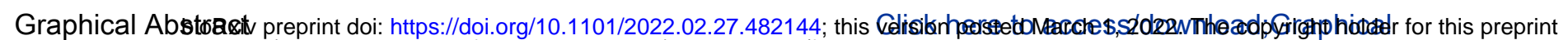

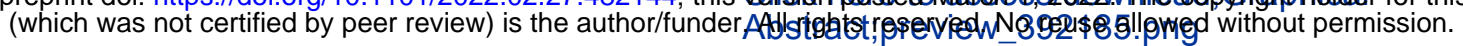
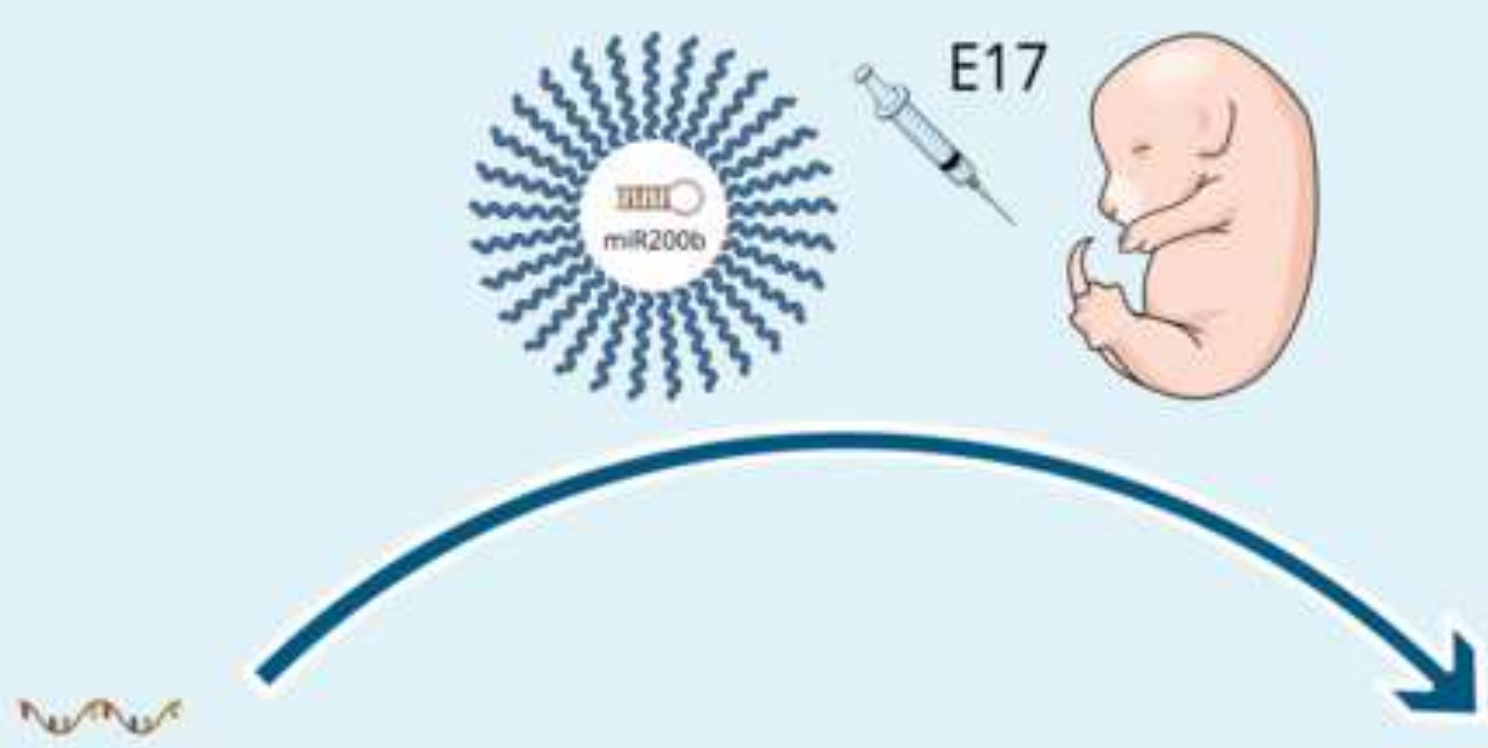

nesters

epigenetic changes

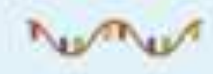

nemes

\author{
lung growth \\ increased airspace
}
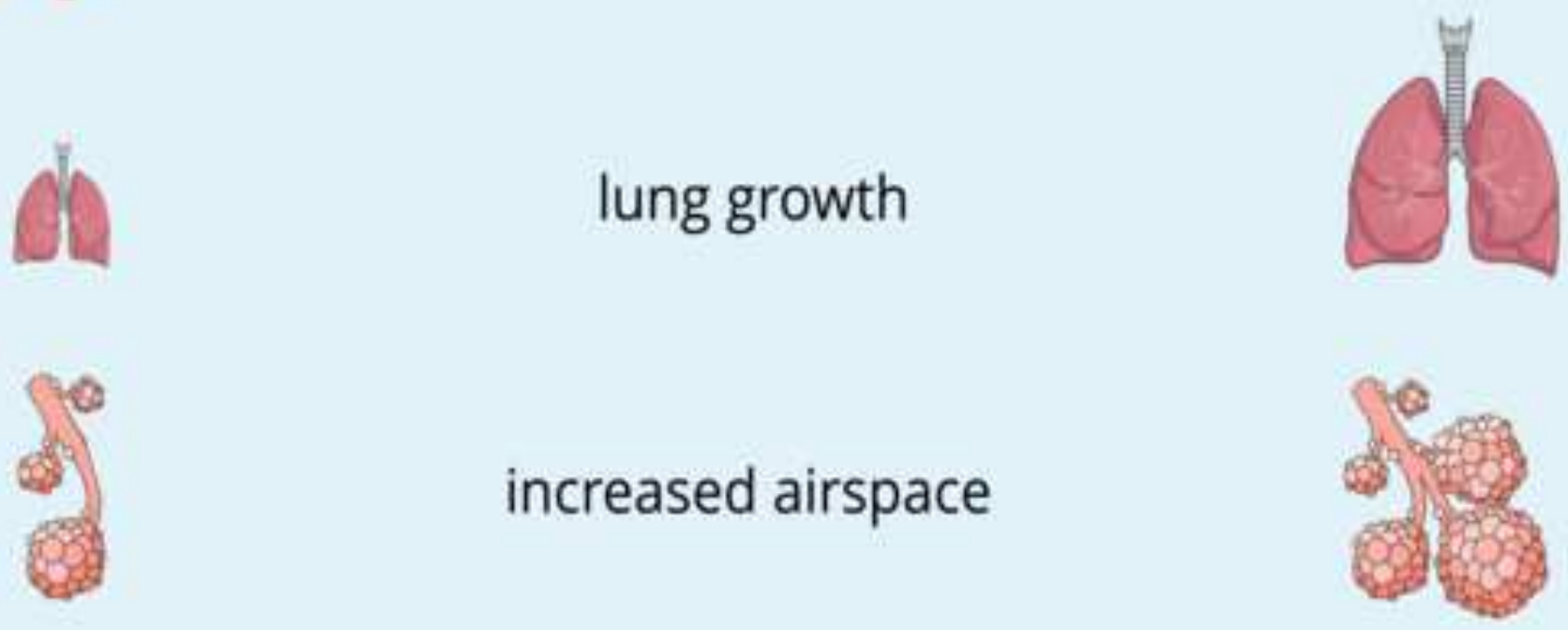

\title{
vascular remodeling
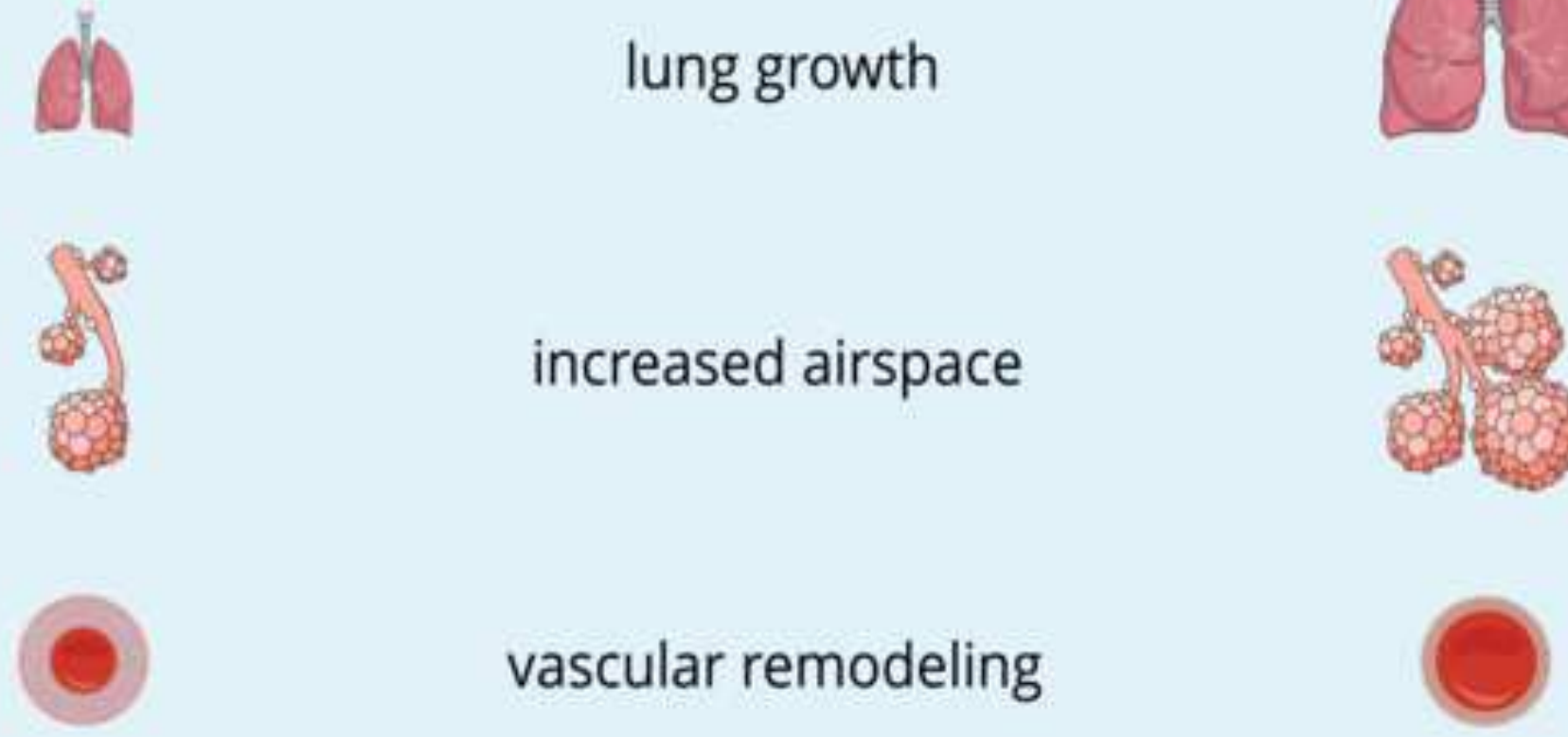